\title{
Influence of different reconstruction parameters in the visualization of intracranial stents using C-arm flat panel CT angiography: experience in an animal model
}

Acta Radiologica

2016, Vol. 57(2) 233-240

(C) The Foundation Acta Radiologica 2015

Reprints and permissions:

sagepub.co.uk/journalsPermissions.nav DOI: $10.1|77 / 0284| 851|557| 988$ acr.sagepub.com

@SAGE

\author{
Sabine Ott*, Tobias Struffert*, Marc Saake, Philipp Gölitz, \\ Edyta Adamek and Arnd Doerfler
}

\begin{abstract}
Background: C-arm flat panel computed tomography angiography (CA-CTA) is a relatively new imaging modality. Consequently, knowledge about postprocessing parameters and their influence on image quality is still limited, especially for the visualization of implanted microstents.

Purpose: To optimize reconstruction parameters by evaluating the influence of these different parameters for CA-CTA visualization of microstents in an animal model.

Material and Methods: Eleven microstents were implanted within the left common carotid artery of I I New Zealand white rabbits. Both CA-CTA, using intra-venous delivery of contrast material, and conventional digital subtraction angiography (DSA) was performed. CA-CTA datasets were reconstructed using three different image characteristics (normal, sharp, smooth). Two experienced neuroradiologists evaluated the image quality and performed measurements of inner and outer stent diameters as well as measurements of the lumen area.

Results: Stent deployment was performed successfully in all animals. Inter-observer correlation coefficient for all measurements was high $(r=0.87-0.92)$. Lumen area and inner stent diameter were significantly smaller in image characteristic "smooth" $(P<0.0 \mathrm{I})$ than in "sharp" and "normal". Outer stent diameter was larger in "smooth" than in "sharp" and "normal" $(P<0.0 \mathrm{I})$. Stent strut size was significantly wider using image characteristic "smooth". "Sharp" and "normal" compared best to DSA, with "sharp" providing the closest match to DSA measurements, with the tradeoff of significantly more noise than in the "normal" reconstructions.

Conclusion: The use of different image characteristics in the postprocessing of CA-CTA datasets has an influence on the visualization of implanted stents. Image characteristic "sharp" and "normal" compared best to DSA.
\end{abstract}

\section{Keywords}

Digital subtraction angiography, C-arm flat panel computed tomography angiography, stent visualization, intracranial stent, animal model

Date received: 4 August 2014; accepted: 18 January 2015

\section{Introduction}

In recent years, $\mathrm{C}$-arm flat panel computed tomography (CA-CT) has been introduced into clinical routine and is increasingly used for a wide variety of imaging tasks. The soft tissue resolution of CA-CT is still limited and has not yet reached the level of conventional multislice CT (MS-CT). On the other hand, the spatial resolution
Department of Neuroradiology, University of Erlangen-Nuremberg, Erlangen, Germany

*Equal contributors.

Corresponding author:

Sabine Ott, Department of Neuroradiology, University of ErlangenNuremberg, Schwabachanlage 6, 91054 Erlangen, Germany. Email: Sabine.Ott@uk-erlangen.de 
has been shown to be superior to MS-CT (1). Taking these characteristics into consideration, CA-CT has been shown to be the superior imaging modality for visualization of small high contrast objects (1-3). In particular, CA-CT could be ideal for imaging small devices such as stents or flow diverters for intracranial use. These devices can be visualized in high resolution to evaluate the shape and placement of the device after deployment. Follow-up imaging is mandatory in patients treated with stents or flow diverters to screen for the occurrence of in-stent restenosis or to confirm aneurysm occlusion. Neither magnetic resonance angiography (MRA) nor multislice CT angiography (MS-CTA) provide reliable data in the visualization of the lumen of intracranial stents compared to the gold standard of digital subtraction angiography (DSA) (4-7).

CA-CT, combined with an injection of intravenous contrast material, has resulted in a new minimally invasive angiographic imaging modality (CA-CTA) and has been shown to detect restenosis after intracranial stenting as well as DSA and superior to MS-CTA $(8,9)$. Similarly, after flow diverter treatment, CA-CTA seems to provide similar information in comparison to DSA (10). As a result of these findings, CA-CTA can be used to support DSA for the follow-up imaging in these patients.

It is well known that the visualization of the stent lumen using MS-CTA imaging is limited due to artificial lumen narrowing (ALN) resulting from blooming artifacts $(1,11,12)$. Blooming artifacts result in stent struts that appear thicker than they really are, and thus impair the visibility of the lumen of a stent. It has been shown that dedicated postprocessing methods may limit blooming artifacts and may improve image quality (13). In MS-CTA, sharp kernels were found to be the best to avoid ALN, whereas medium kernels seem to be most suitable to recognize in-stent re-stenosis (14).

CA-CTA postprocessing software used at our institution (InSpace 3D, Siemens AG Healthcare Sector, Forchheim, Germany) allows for the application of different postprocessing parameters (so-called "image characteristics") analogous to MS-CT kernels. As a new imaging modality, knowledge regarding the influence of these different "image characteristics" on the image quality of CA-CTA is unknown, but may have an influence on the visualization of implanted devices. The aim of our study was to compare different image characteristics applied during CA-CTA reconstruction in order to evaluate the influence on the visualization of stents. Our hypothesis was that ALN due to blooming artifacts may also be present in CA-CTA and may be optimized by selection of appropriate image characteristic parameters to achieve best possible image quality for evaluation of the lumen of stents in comparison to DSA.

\section{Material and Methods}

\section{Animal model and imaging}

The study was approved by the local Animal Protection Committee. An animal model based on New Zealand White rabbit (4-5 kg body weight) was used as previously described (8). The common carotid artery of these rabbits has a diameter in the range of $2.5-3 \mathrm{~mm}$, an identical diameter range to human intracranial arteries. All animals received Aspirin and Clopidogrel, dissolved in drinking water, as an anticoagulation therapy 3 days before stent placement. At the start of the procedure, the right femoral artery was surgically exposed under general anesthesia to gain arterial access. The femoral artery was distally ligated and a $4 \mathrm{~F}$ introducer sheath (Radiofocus Introducer II, Terumo Cooperation, Terumo Europe N. V., Leuven, Belgium) was placed. Using standard angiographic techniques, a microcatheter (Tracker Excel 14, Boston Scientific/ Target, Fremont, CA, USA) was advanced to the left common carotid artery. Selective DSA was performed to visualize the anatomy, and then an exchange wire (Synchro Standard; Boston Scientific/ Target, Fremont, CA, USA) was placed. Using this access, six self-expandable (Wingspan ${ }^{\mathrm{TM}}$; Boston Scientific/Target, Fremont, CA, USA) and five balloon-expandable stents (Micro Driver, Medtronic, Galway, Ireland) with the size of $3-3.5 \mathrm{~mm}$ in diameter and $15-30 \mathrm{~mm}$ in length, were implanted. Stent deployment was restricted to only one device per carotid artery to avoid any possible interaction. After stent deployment another DSA series was obtained. Next, the microcatheter and introducer sheath were removed. The femoral vein was then exposed and a puncture was performed using a standard venous 22G catheter (Vasofix Safety, Braun Melsungen AG, Melsungen, Germany) to gain venous access for CACTA imaging (8). Immediately following imaging, the animals were sacrificed.

\section{DSA and CA-CTA imaging}

DSA and CA-CTA were performed using a flat detector equipped angiographic system (Axiom Artis dBA, Siemens AG, Healthcare Sector, Forchheim, Germany).

A standard DSA program (4 frames/s; dose, $3 \mu \mathrm{Gy}$ per frame; field of view [FOV], $11 \mathrm{~cm}$ ) was used. Each DSA sequence was performed using a manual injection of contrast material (Imeron 300, Bracco Imaging, 
Konstanz, Germany) using a $1 \mathrm{~mL}$ syringe with a total volume of $0.5 \mathrm{~mL}$.

A standard CA-CT application (10 s DSA program, as provided by the manufacturer) was used for CA-CTA imaging. This program is characterized by the following parameters: rotation time, $10 \mathrm{~s}$; detector size, $30 \times 40 \mathrm{~cm}$; angular range, $200^{\circ}$; increment, $0.8^{\circ} /$ image; dose, $1.2 \mu \mathrm{Gy} /$ frame; and tube voltage, $70 \mathrm{kV}$. As described by others we used $1 \mathrm{~mL}$ contrast material (Imeron 300) per $1 \mathrm{~kg}$ body weight, followed by $6 \mathrm{~mL}$ saline flush. Injection rate was $1 \mathrm{~mL} / \mathrm{s}$ both for contrast and saline (8). Injection was performed using a dual syringe power injector (Accutron HP-D, MEDTRON, Saarbrücken, Germany). In order to achieve consistent arterial opacification, a bolus watching method was used (15).

\section{Postprocessing}

Angiographic images and CA-CTA datasets were postprocessed using standard software as provided by the manufacturer using a dedicated workstation (InSpace 3D and Angio software, Siemens AG Healthcare Sector, Forchheim, Germany). The DSA images were subtracted and pixel shift was used to achieve the best possible DSA image quality. The InSpace 3D software was used to reconstruct the CA-CTA datasets. This software includes standard automatic applications of system-specific filter algorithms to correct for beam hardening, scattered radiation, and ring artifacts. The software allows for the application of different postprocessing parameters that can be chosen by the investigator. Initially, a so-called "kernel" can be chosen. We used setting "HU" (abbreviation for "Hounsfield") which is recommended by the manufacturer to postprocess multiplanar reconstructions (MPR). The alternative setting "EE" (abbreviation for "edge enhancement") is recommended by the manufacturer to reconstruct subtracted three-dimensional rotational angiography datasets with intra-arterial contrast application and was therefore not used. The next parameter was the subject of our study and is called "image characteristics". This parameter is analogous to MSCT kernels and the possible settings of "sharp", "normal", and "smooth" were used for comparison. The reconstruction resulted in an isotropic volume dataset with a batch of 450 slices in a $512 \times 512$ matrix. Single slice thickness of this batch of images was $0.1 \mathrm{~mm}$ and FOV of $80 \mathrm{~mm}$. Multiplanar reformats were created with $1 \mathrm{~mm}$ slice thickness and $0.5 \mathrm{~mm}$ spacing for both the axial and coronal planes (16). Images were anonymized, randomized, and transferred to a workstation for evaluation. Reviewers were allowed to adjust the windowing as desired. A mean window center of 1000 and a window width of 3500 were used for all images.

\section{Imaging analysis}

In consensus, two experienced neuroradiologists performed a subjective review of the images (DSA, CA-CTA) according to the following grading scales. The measurements were performed separately by each neuroradiologist so that a statistical inter-observer comparison was possible.

DSA images were evaluated using the following grading scale: 1, perfect quality (homogenous opacification of the stent lumen, sharp delineation of the artery, no movement artifacts); 2, acceptable quality (acceptable opacification, good delineation of the artery, only minimal movement artifacts); and 3 , poor image quality (inhomogenous opacification, poor delineation of the artery, obvious movement artifacts).

The visualization of the stent lumen on CA-CTA images was evaluated with a subjective 3-point scale for diagnostic quality. The reviewers evaluated if the visualization of the stent lumen was: 1, highly opacified as compared to surrounding tissue; 2, contrast visible and suitable for diagnostic evaluation; or 3 , no contrast visible and therefore not of diagnostic value.

Image noise was evaluated with a subjective 4-point scale for diagnostic quality. The reviewers evaluated if: 1 , no image noise was visible; 2 , image noise was visible, but acceptable; 3 , image noise was visible, but images are impaired; or 4 , image noise was massive, image not of diagnostic quality.

Stents for intracranial use have very thin struts that are hardly recognizable on DSA images, while in CA-CTA an outer and inner diameter is visible. The reviewers were asked to measure the diameter on DSA images. This value served as the gold standard of stent lumen diameter to be compared to inner diameter in CA-CTA.

In CA-CTA stent struts are visible meaning that an inner and outer diameter of the stent can be recognized. Due to blooming, the stent struts may appear thicker than they are. This blooming artifact may depend on the image characteristic used for postprocessing. The reviewers were asked to measure the assumed inner diameter, representing the stent lumen, and the assumed outer diameter on CA-CTA images as shown on Fig. 1a-c. Furthermore, lumen area and total stent area including stent struts were measured as shown on Fig. 1d and e. The difference of these values was calculated, summed up, and the mean value calculated (Table 1). All measurements were done using standard tools on the Leonardo workstation.

\section{Statistical analysis}

Measurements by both neuroradiologists were compared by Pearson correlation coefficient. The mean value of each pair of measurements was calculated 


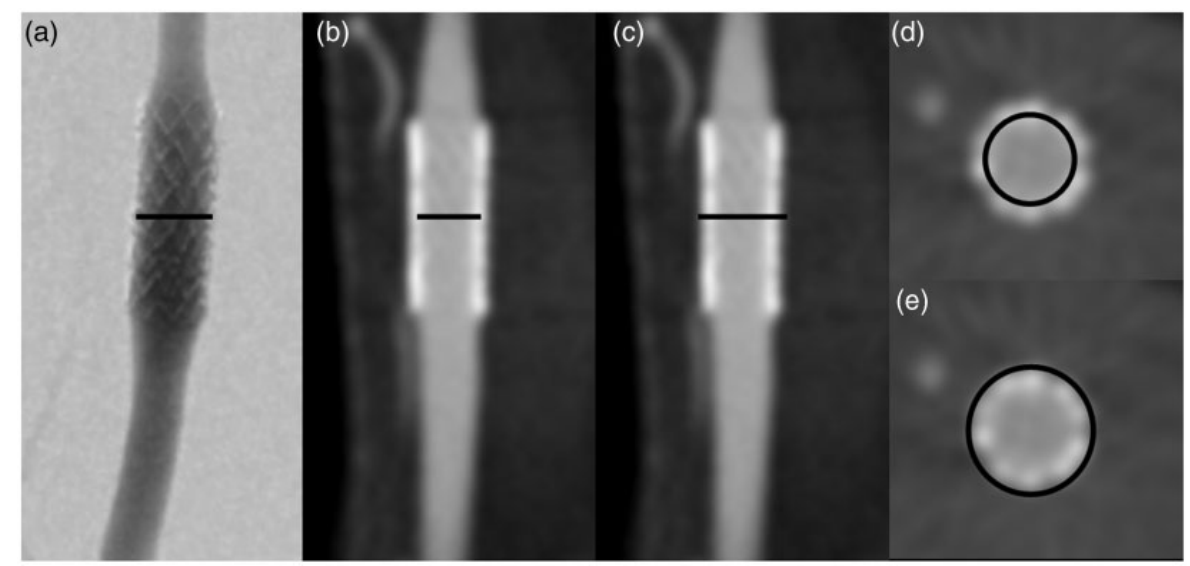

Fig. I. Measurements were performed as shown above: Stent diameter in DSA (a), inner stent diameter in CA-CTA (b), outer stent diameter in CA-CTA (c), lumen area in CA-CTA (d), and total stent area in CA-CTA (e).

Table I. Averaged readings of the two neuroradiologists, measurements of stent lumen and stent size as area values performed as shown in Fig. Id and e.

\begin{tabular}{|c|c|c|c|c|c|c|c|c|c|}
\hline \multirow[b]{2}{*}{ Stent } & \multicolumn{3}{|c|}{ Lumen area in $\mathrm{cm}^{2}$} & \multicolumn{3}{|c|}{ Total stent area in $\mathrm{cm}^{2}$} & \multicolumn{3}{|c|}{ Difference in $\mathrm{cm}^{2}$} \\
\hline & Sharp & Normal & Smooth & Sharp & Normal & Smooth & Sharp & Normal & Smooth \\
\hline 1 & 0.055 & 0.050 & 0.035 & 0.080 & 0.090 & 0.060 & 0.025 & 0.040 & 0.025 \\
\hline 2 & 0.050 & 0.035 & 0.030 & 0.090 & 0.095 & 0.105 & 0.040 & 0.060 & 0.075 \\
\hline 3 & 0.035 & 0.030 & 0.030 & 0.070 & 0.070 & 0.075 & 0.035 & 0.040 & 0.045 \\
\hline 4 & 0.050 & 0.045 & 0.045 & 0.095 & 0.095 & 0.115 & 0.045 & 0.050 & 0.070 \\
\hline 5 & 0.050 & 0.045 & 0.030 & 0.095 & 0.100 & 0.110 & 0.04 & 0.055 & 0.080 \\
\hline 6 & 0.055 & 0.050 & 0.045 & 0.100 & 0.095 & 0.105 & 0.045 & 0.045 & 0.060 \\
\hline 7 & 0.055 & 0.050 & 0.045 & 0.100 & 0.100 & 0.100 & 0.045 & 0.050 & 0.055 \\
\hline 8 & 0.090 & 0.090 & 0.075 & 0.150 & 0.165 & 0.160 & 0.060 & 0.075 & 0.085 \\
\hline 9 & 0.045 & 0.045 & 0.035 & 0.095 & 0.090 & 0.095 & 0.050 & 0.045 & 0.060 \\
\hline 10 & 0.045 & 0.040 & 0.035 & 0.085 & 0.095 & 0.100 & 0.040 & 0.055 & 0.065 \\
\hline \multirow[t]{3}{*}{11} & 0.040 & 0.035 & 0.025 & 0.070 & 0.070 & 0.070 & 0.030 & 0.035 & 0.045 \\
\hline & & & & & & & \multicolumn{3}{|c|}{ Average stent area in $\mathrm{cm}^{2}$} \\
\hline & & & & & & & 0.04 & 0.05 & 0.06 \\
\hline
\end{tabular}

and used for further statistical analysis of Pearson Correlation. Statistical significance was assumed for $P$ values $<0.01$. All statistics were performed using SPSS 18.0 (SPSS Inc., Chicago, IL, USA).

For calculation of ALN a formula as proposed by Hähnel et al. was used (11):

ALN $=\left[1-\left(\right.\right.$ Diameter CA-CTA $A_{\text {sharp } / \text { normal } / \text { smooth } /}$ Diameter DSA)]* $100 \%$. The CA-CTA values for the inner diameter were used in this calculation.

\section{Results}

Stents were implanted successfully in all animal subjects. CA-CTA datasets were reconstructed using three different image characteristics (sharp, normal, smooth) and were postprocessed in axial and coronal planes as MPR images. All DSA and CA-CTA images were rated by both reviewers to be grade 1 on the subjective evaluation scale so that further evaluation was possible in all subjects. All CA-CTA images reconstructed with image characteristic "smooth" were rated as grade 1 for the image noise evaluation. Images reconstructed by "normal" and "sharp" were grade 2 and 3, respectively, for image noise. The reviewers noticed beam (metal) hardening artifacts due to the markers of the Wingspan stent.

Since the size of the stent struts of the Wingspan $(0.0030$ inches equaling $0.1 \mathrm{~mm})$ and the Micro Driver $(0.0036$ inches equaling $0.1 \mathrm{~mm})$ stent systems are nearly identical, the measurements of both stent systems were summed up and not evaluated separately. The Pearson's correlation coefficients in inter-observer 


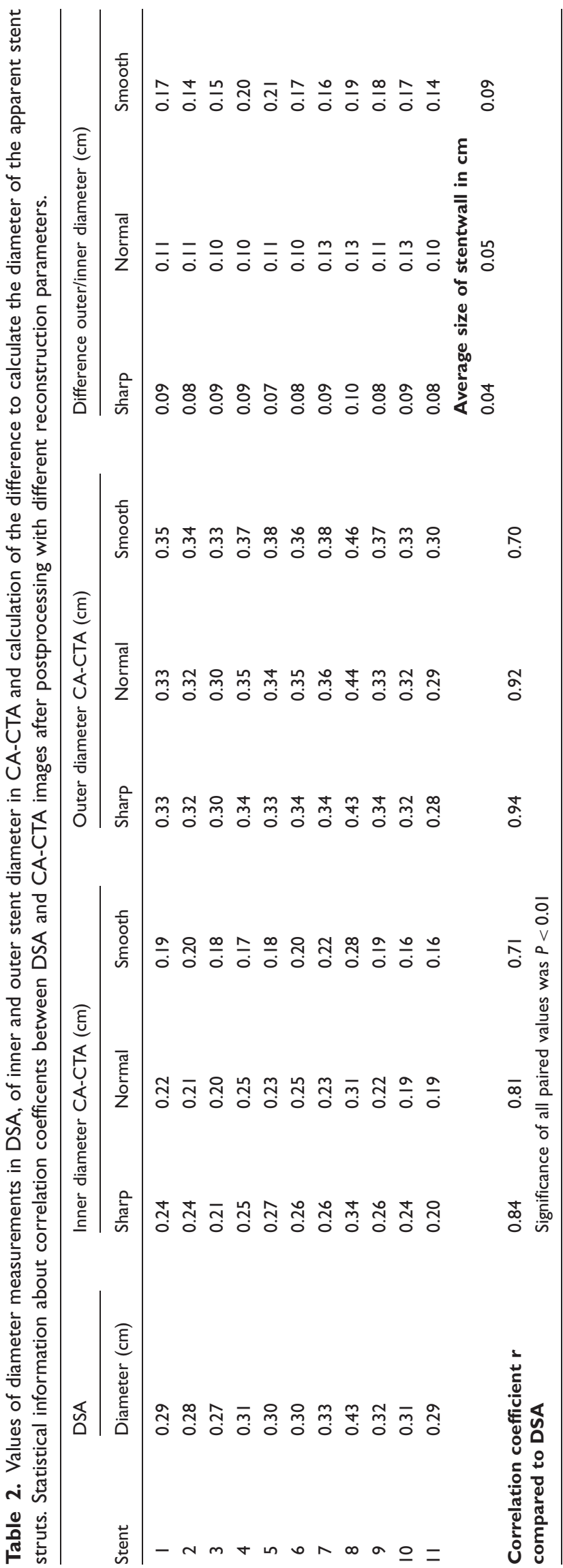

measurements were high in image characteristics sharp and normal $(r=0.87-0.92)$. The inter-observer correlation in image characteristic "smooth" was in the range of $0.74-0.81$. Due to this significantly high agreement, the values were averaged for further statistical analysis.

Diameter measurements were performed in the middle part of stents (Fig. 1a-c). In DSA, different measurements of inner stent diameter representing the lumen and outer stent diameter are not possible. Despite this, DSA values were compared to inner and outer stent diameter of CA-CTA images. All values are shown in Table 2.

There was a high correlation between parameter "sharp" $(r=0.94)$ and "normal" $(r=0.92)$ compared to DSA in terms of measurement of outer diameter. Correlation to inner lumen diameter was also high for "sharp" ( $\mathrm{r}=0.84)$ and "normal" $(\mathrm{r}=0.81)$. Image characteristic "smooth" showed less correlation compared to DSA, $r=0.71$ for inner diameter and $r=0.7$ for outer diameter. The Pearson's test resulted in statistically significant $P<0.01$ for all paired values (Table 2). The difference of the inner and outer diameter was calculated, summed up, and the mean value divided by 2 was calculated to represent the stent strut size. In "sharp" and "normal" the size was 0.4 and $0.5 \mathrm{~mm}$, respectively, and with both resulting in diameter measurements of the struts appearing four times larger than they really are. This was even more pronounced in "smooth" with a size of $0.9 \mathrm{~mm}$ (nine times larger).

Stent lumen and struts could be identified most clearly in "sharp" reformatted images (Fig. 2b and f). Stent struts can also be recognized in "normal" postprocessed images (Fig. 2c and g) whereas struts are not delineated in "smooth" images (Fig. 2d and h). Subjectively, there was a better delineation of the contrast enhanced lumen in "sharp" and "normal" postprocessed images than in "smooth".

To quantify the ALN, a formula as described by Hähnel et al. (11) was used. The inner stent lumen was compared to DSA and is expressed in percentages. The averaged ALN was $21 \%$ for "sharp" reformatted images and $29 \%$ for "normal" reformatted images. An averaged ALN of $41 \%$ was seen on "smooth" reformatted images.

In axial reconstructed MPR images the enhanced stent lumen could also be recognized significantly better in "sharp" (Fig. 3a and d) and "normal" (Fig. 3b and e) postprocessed images than in "smooth" (Fig. 3c and f). Stents struts were best seen in "sharp" (Fig. 3a and d), but image noise became more significant (Fig. 3a). The ALN effect was nearly identical in image characteristic "normal" but image noise was less visible than in "sharp" (Fig. 3b and e). Image characteristic "smooth" resulted in stents struts that were blurred like a ring structure (Fig. 3c and f). 


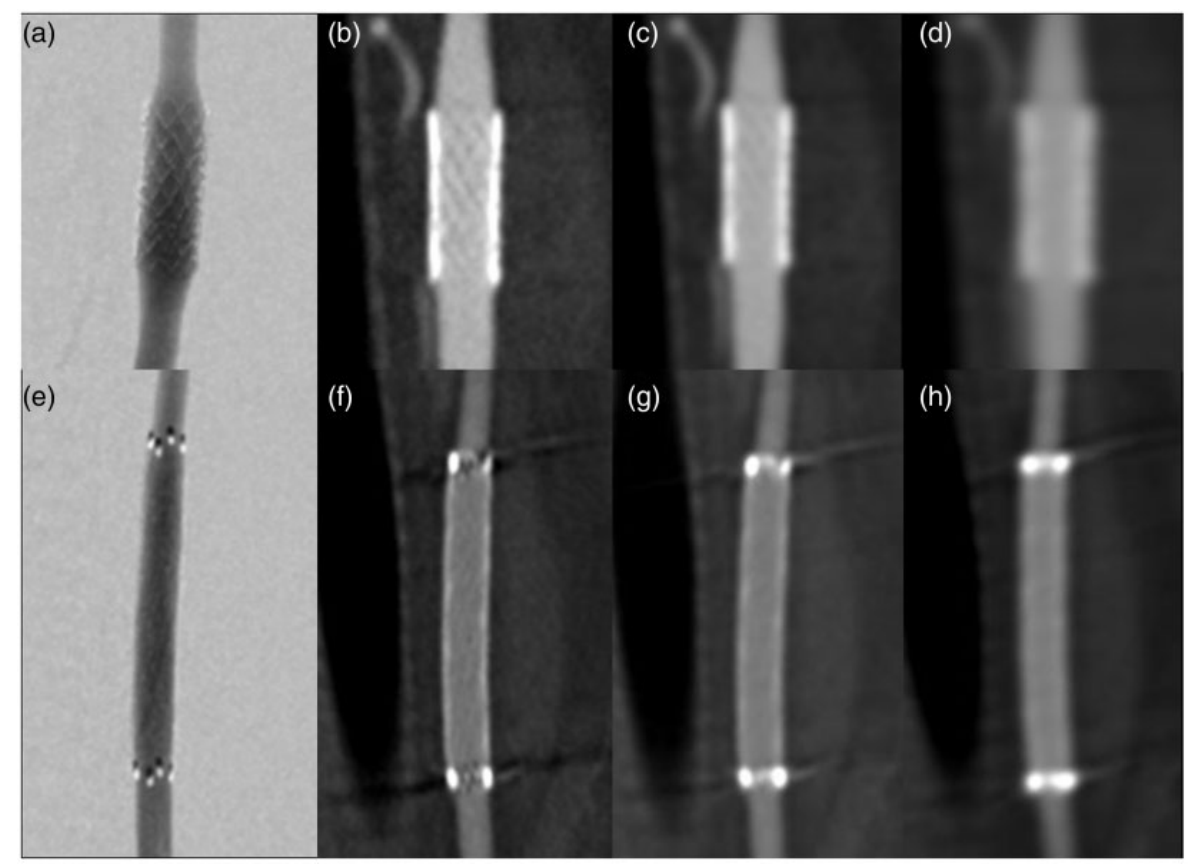

Fig. 2. Visualization of Micro-Driver (a-d) and Wingspan (e, $f$ ) stent system in DSA and CA-CTA. DSA (a, e), coronal reconstructed images of CA-CTA image characteristic "sharp" (b, f), CA-CTA image characteristic "normal" (c, g), CA-CTA image characteristic "smooth" ( $d, h)$. There is an increase of image noise from "smooth" to "sharp". In (b) and (f) the lumen of the stents appears noisy while in (c) and (g) the lumen appears homogenous. Beam hardening artifacts of the stent markers of the Wingspan stent system are visible.

\section{Discussion}

Follow-up imaging of intracranial stents is challenging. Though DSA serves as the gold-standard, a minimally invasive method is highly desirable. For re-assessment after intracranial stenting, precise imaging of the stent lumen is necessary in order to diagnose possible re-stenosis. Different minimal invasive imaging modalities have been evaluated to recognize in-stent re-stenosis. Magnetic resonance imaging (MRI) appears to be promising (17). However, MRI is challenging in the visualization of stents due to the artifacts caused by the interaction of the device materials with the magnetic field and radiowaves used in MRI. This interaction may result in a significant signal-intensity loss within the lumen and adjacent to the stent, most likely related to stent-induced radio-frequency shielding (4-7). Therefore MRI is currently not accepted as a followup imaging method for intracranial stenting.

The use of MS-CTA in the visualization of stents is impaired by ALN. In vitro studies of small vessel stents demonstrated that the minimum diameter of stents for reliable visualization of the lumen using MS-CTA was $4.0 \mathrm{~mm}(1,11,12)$. There are only small case series in patients available that seem to confirm the results of these in vitro studies (9).

Recently, CA-CTA has been introduced as a new non-invasive high spatial resolution imaging modality with several different applications. Specifically, this new method seems to be promising for the visualization of intracranial stents. In an animal model, CA-CTA correlated well with the visualization of stents compared to DSA, while conventional MS-CTA failed to visualize the lumen of the stent device (8). In a phantom study and a small case series, Psychogios et al. demonstrated that CA-CTA was comparable to DSA in the recognition and quantification of in-stent re-stenosis $(9,18)$. These promising findings in patients were confirmed by others using an optimized image protocol (15).

Because CA-CTA is a relatively new imaging modality, limited information is available regarding the optimization of reconstruction parameters for a given imaging task, such as intra-cranial stent visualization. It is well known that ALN is impairs CTA images of small vessel stents. Additionally, it is well known that different kernels applied during post-processing may at least limit this problem. It was our hypothesis that ALN may also be present in CACTA, and similar to CTA, may be optimized by appropriate postprocessing parameter selection that are supported by the software. These parameters for CA-CTA are called "image characteristic" and are analogous to kernels in CT. This paper provides first results for the visualization of stents for intracranial use with different postprocessing parameters. Our results indicate that ALN is also present in CA-CTA and is dependent on the postprocessing parameters 


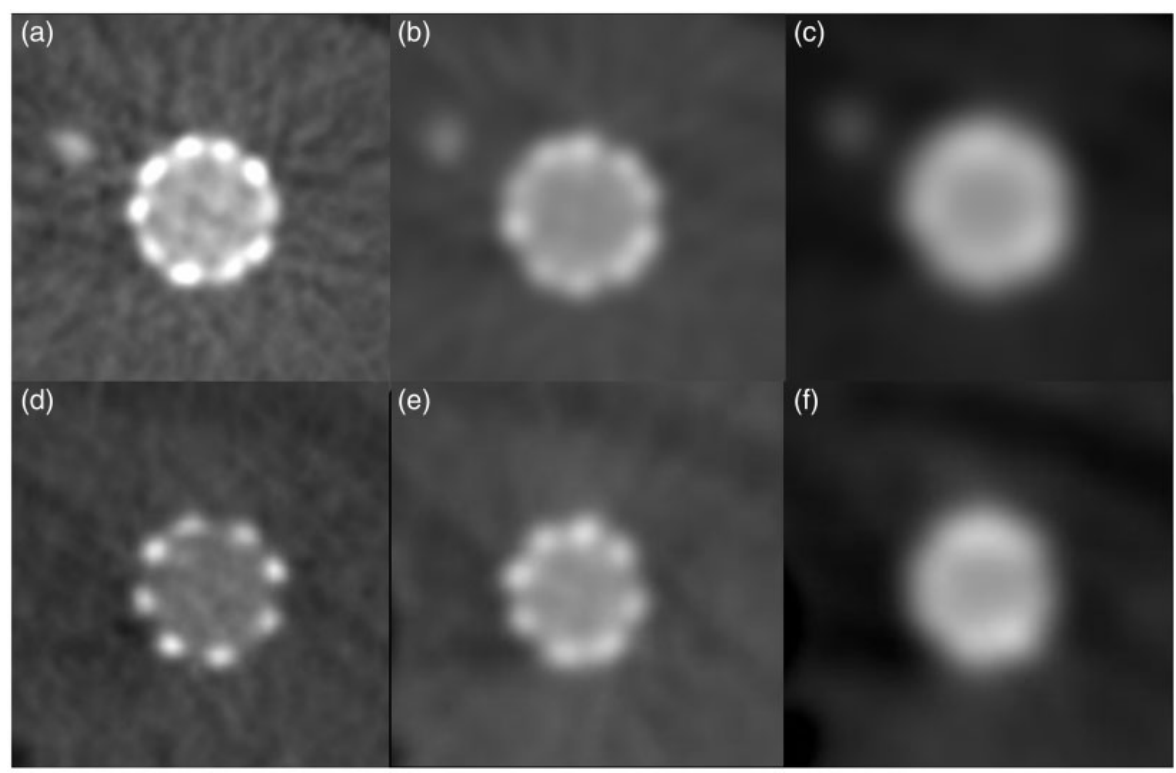

Fig. 3. Axial reconstructed CA-CTA datasets. (a-c) Micro-Driver; (d-f) Wingspan. Image characteristic "sharp" (a, d), "normal" $(b, e)$, and "smooth" (c, f). There is an increase of image noise from "smooth" to "sharp". The lumen in images (c) and (f) reconstructed by "smooth" is poorly defined and blurred.

used. The "smooth" parameter is not useful in the visualization of stents and results in struts that are merged to a ring like structure and the lumen becomes blurred by ALN. The "sharp" parameter provided best correlation to DSA. On the other hand, image noise was most obvious in these images in comparison to images postprocessed by the "normal" and "smooth" parameters. This image noise could potentially interfere with the visualization of thrombotic material within the lumen of the stent. In our opinion, the "normal" parameter offered the best compromise between ALN and image noise.

Our study is limited by the small number of samples, but is sufficient to show that the ALN effect does depend on the postprocessing parameter. Future investigations of stents exhibiting in-stent re-stenosis are necessary in order to further evaluate the performance of different postprocessing parameters on the clinical in vivo visualization of in-stent material representing re-stenosis. One method to reduce the image noise for CA-CTA is to increase the radiation dose of the acquisition program. Unfortunately, this hypothesis could not be evaluated in the present study as the modification of X-ray dose for a given CA-CT procedure is not permitted with commercial CA-CT systems without special calibration. As a future project, CA-CT programs with different acquisition parameters (e.g. dose, number of frames, tube voltage) should be implemented and be compared. This may further optimize and increase the knowledge about image quality in CA-CT. In addition to image noise, beam hardening (metal) artifacts were apparent due to the markers of the Wingspan stent. Similar results have been reported by other groups (15). Initial results of prototype software used to reduce these artifacts indicate that methods may be available to correct for these issues in the near future $(19,20)$. While the research of this study did not include these correction techniques, future work should be done to incorporate these new methods in conjunction with optimization of postprocessing parameters and acquisition protocols.

In conclusion, image characteristic parameters used in the reconstruction of CA-CTA images have a significant influence on the visualization of the struts and the lumen of the stents. Image characteristic "sharp" was shown to provide the best correlation to DSA, showing the least ALN. ALN for image characteristic "normal" marginally increased ALN but with the significant advantage that image noise is greatly reduced. Therefore, we recommend "normal" to be the best compromise of ALN minimization and overall image quality. Image characteristic "smooth" is not suitable in the visualization of stents.

\section{Acknowledgments}

The authors gratefully thank Stryker Neurovascular, Fremont CA, USA, for kindly providing Wingspan stents, microcatheters, and wires. Additionally, the authors acknowledge funding of the Medical Valley national leading edge cluster, Erlangen, Germany, diagnostic imaging network, sub-project BD 16, research grant no. 13EX1212G.

\section{Declaration of interest}

The authors report no conflicts of interest. The authors alone are responsible for the content and writing of the paper. 


\section{Funding}

This research received no specific grant from any funding agency in the public, commercial, or not-for-profit sectors.

\section{Research ethics}

As required by German law, the study was approved by the animal protection committee of the government of Mittelfranken (91511 Ansbach), study approval number 542531.31-20/07 issued by 22.11.2007.

\section{References}

1. Ionescu M, Metcalfe RW, Cody D, et al. Spatial resolution limits of multislice computed tomography (MS-CT), Carm-CT, and flat panel-CT (FP-CT) compared to MicroCT for visualization of a small metallic stent. Acad Radiol 2011;18:866-875.

2. Struffert T, Saake M, Ott S, et al. Intravenous flat detector $\mathrm{CT}$ angiography for non-invasive visualisation of intracranial flow diverter: technical feasibility. Eur Radiol 2011;21: 1797-1801.

3. Benndorf G, Strother CM, Claus B, et al. Angiographic CT in cerebrovascular stenting. Am J Neuroradiol 2005; 26:1813-1818.

4. Golshani B, Lazzaro MA, Raslau F, et al. Surveillance imaging after intracranial stent implantation: non-invasive imaging compared with digital subtraction angiography. J Neurointerv Surg 2013;5:361-365.

5. van Holten J, Wielopolski P, Bruck E, et al. High flip angle imaging of metallic stents: implications for MR angiography and intraluminal signal interpretation. Magn Reson Med 2003;50:879-883.

6. Lovblad KO, Yilmaz H, Chouiter A, San Millan Ruiz D, et al. Intracranial aneurysm stenting: follow-up with MR angiography. J Magn Reson Imaging 2006;24:418-422.

7. Seok JH, Choi HS, Jung SL, et al. Artificial luminal narrowing on contrast-enhanced magnetic resonance angiograms on an occasion of stent-assisted coiling of intracranial aneurysm: in vitro comparison using two different stents with variable imaging parameters. Korean J Radiol 2012;13:550-556.

8. Struffert T, Ott S, Adamek E, et al. Flat-detector computed tomography in the assessment of intracranial stents: comparison with multi detector CT and conventional angiography in a new animal model. Eur Radiol 2011;21: 1779-1787.

9. Psychogios MN, Schramm P, Buhk JH, et al. Angiographic CT after intravenous contrast agent application: A noninvasive follow-up tool after intracranial angioplasty and stenting. Am J Neuroradiol 2010;31: 1886-1891.

10. Saake M, Struffert T, Goelitz P, et al. Angiographic CT with intravenous contrast agent application for monitoring of intracranial flow diverting stents. Neuroradiology 2012;54:727-735.

11. Hahnel S, Trossbach M, Braun C, et al. Small-vessel stents for intracranial angioplasty: in vitro comparison of different stent designs and sizes by using CT angiography. Am J Neuroradiol 2003;24:1512-1516.

12. Trossbach M, Hartmann M, Braun C, et al. Small vessel stents for intracranial angioplasty: in vitro evaluation of in-stent stenoses using CT angiography. Neuroradiology 2004;46:459-463.

13. Maintz D, Seifarth H, Flohr T, et al. Improved coronary artery stent visualization and in-stent stenosis detection using 16-slice computed-tomography and dedicated image reconstruction technique. Invest Radiol 2003;38: 790-795.

14. Jang JH, Lim YS, Lee YJ, et al. Evaluation of middle cerebral artery stents using multidetector row CT angiography in vivo study: comparison of the three different kernels. Acta Radiol 2012;53:456-460.

15. Struffert T, Kloska S, Engelhorn T, et al. Optimized intravenous Flat Detector CT for non-invasive visualization of intracranial stents: first results. Eur Radiol 2011; 21:411-418.

16. Struffert T, Doelken M, Adamek E, et al. Flat-detector computed tomography with intravenous contrast material application in experimental aneurysms: comparison with multislice CT and conventional angiography. Acta Radiol 2010;51:431-437.

17. Prabhakaran S, Warrior L, Wells KR, et al. The utility of quantitative magnetic resonance angiography in the assessment of intracranial in-stent stenosis. Stroke 2009; 40:991-993.

18. Psychogios MN, Schramm $\mathrm{P}$, Amelung $\mathrm{N}$, et al. Evaluation of Noninvasive Follow-up Methods for the Detection of Intracranial In-Stent Restenosis: A Phantom Study. Invest Radiol 2013;48:98-103.

19. Prell D, Kyriakou Y, Beister M, et al. A novel forward projection-based metal artifact reduction method for flatdetector computed tomography. Phys Med Biol 2009; 7546575-6591.

20. Prell D, Kyriakou Y, Struffert T, et al. Metal artifact reduction for clipping and coiling in interventional Carm CT. Am J Neuroradio 2010;31:634-639. 